\title{
Expression profile analysis of 9 heat shock protein genes throughout the life cycle and under abiotic stress in rice
}

\author{
YE ShuiFeng $^{1,2}$, YU ShunWu ${ }^{1}$, SHU LieBo $^{1}$, WU JinHong ${ }^{1}$, WU AiZhong ${ }^{2,3}$ \& LUO LiJun ${ }^{1 *}$ \\ ${ }^{1}$ Shanghai Agrobiological Gene Center, Shanghai 201106, China; \\ ${ }^{2}$ School of Agriculture and Biology, Shanghai Jiaotong University, Shanghai 201101, China; \\ ${ }^{3}$ Shanghai Academy of Agricultural Sciences, Shanghai 201106, China
}

Received June 22, 2011; accepted August 16, 2011

\begin{abstract}
Plant heat shock proteins (Hsps) facilitate protein folding or assembly under diverse developmental and adverse environmental conditions. Nine OsHsps were identified in our previous study from a proteomic analysis of rice cv. IRAT 109 leaf samples at the seedling stage under drought stress. To obtain additional information on the $9 \mathrm{OsHsp}$ genes, this study focused on an expression profile analysis at different development stages throughout the life cycle of rice, and under different abiotic stresses and phytohormone treatments. The 9 genes exhibited distinctive expression patterns in different organs or development stages. Five of the genes (OsHsp72.90, OsHsp72.57, OsHsp71.18, OsHsp24.15 and OsHsp18.03) showed high expression in the endosperm, indicating that $O s H s p$ genes may play important roles in rice seed development. All 9 OsHsps were up-regulated under heat, polyethylene glycol, and abscisic acid treatment, whereas salt stress caused up-regulation of 6 genes (OsHsp93.04, OsHsp 71.10 , OsHsp71.18, OsHsp72.57, OsHsp24.15 and OsHsp18.03) and cold stress resulted in down-regulation of OsHsp93.04 and OsHsp72.57. These diverse expression profiles imply potential functional diversity of the Hsp gene family in rice.
\end{abstract}

heat shock protein, drought, heat, real-time PCR, rice

Citation: Ye S F, Yu S W, Shu L B, et al. Expression profile analysis of 9 heat shock protein genes throughout the life cycle and under abiotic stress in rice. Chin Sci Bull, 2012, 57: 336-343, doi: 10.1007/s11434-011-4863-7

Plants are frequently challenged by persistently changing stress factors such as drought, salinity, cold, and high temperatures. These abiotic stresses usually cause protein dysfunction. Maintenance of proteins in their functional conformations and prevention of the aggregation of non-native proteins are particularly important for cell survival under stress. Heat-shock proteins (Hsps) function as molecular chaperones that assist protein folding or assembly and prevent irreversible protein aggregation to maintain cellular homeostasis under both optimal and adverse developmental conditions [1].

Based on their approximate molecular weights, 5 major families of Hsps are recognized: Hsp100, Hsp90, Hsp70, Hsp60, and the small Hsp (sHsp) families. The Hsp100 ranges in molecular weight from 100 to $104 \mathrm{kD}$, which is a

*Corresponding author (email: lijun@sagc.org.cn) major heat-regulated protein family in diverse organisms that interacts transiently with non-native protein species and prevents the aggregation and promotion of correct folding of proteins. Hsp101 was assumed to play a pivotal role in heat tolerance in Arabidopsis thaliana. Transgenic Arabidopsis plants expressing lower amounts of Hsp101 showed a severely diminished capacity to acquire heat tolerance after mild conditioning pretreatments [2].

The Hsps in the molecular weight range of 82 to $90 \mathrm{kD}$ are classified as the Hsp90 family. These proteins have been isolated and cloned from many plant species. A rice (Oryza sativa) Hsp90 plays an important role in salt stress [3]. In Arabidopsis, AtHsp90s are essential for tolerance to biotic and abiotic stresses [4,5]. AtHsp90.3 was shown to confer heat tolerance in Saccharomyces cerevisae, but resulted in heat sensitivity in transgenic Arabidopsis plants [6]. PgHsp90 from Pennisetum glaucum was indicated to play 
an adaptive or protective role to counter stress-induced protein damage [7].

The Hsp70 family represents one of the most highly conserved classes of heat shock proteins and has molecular weights in the range of 68 to $75 \mathrm{kD}$. At least 18 genes encode Hsp70 proteins in Arabidopsis [8]. Cytosolic Hsp70 plays important roles under abiotic and biotic stresses [9]. AtHsp70s in the plastid stroma are important for thermotolerance of germinating seeds, which indicates that plastid physiology is important for seeds to endure heat stress [10]. In addition, several researchers showed that the Hsp70 family in other plant species is involved in plant development and tolerance to a variety of stresses [1,11-14].

The proteins of the Hsp60 family vary in size from 58 to $65 \mathrm{kD}$. It is generally agreed these proteins are important for assistance of plastid proteins such as Rubisco, and bind different proteins after their transcription and before folding to prevent aggregation of the proteins [15].

The small heat shock proteins (sHsps) include proteins with molecular weights in the range of $15-42 \mathrm{kD}$ [16]. The sHsps can bind to partially folded or denatured proteins, which prevents irreversible unfolding or incorrect protein aggregation, or binds to unfolded proteins and allows further refolding by Hsp70/Hsp100 complexes [15]. Cloning and function analysis of sHsps have been performed in a variety of plant species. In rice, higher expression levels of sHsp17.7 were recorded in sHsp17.7 transgenic plants. A drought tolerance experiment showed overproduction of sHsp17.7 could increase drought tolerance in transgenic rice seedlings [17].

Rice is the main staple food for a large segment of the world's population and the productivity of rice is restricted by a broad range of environmental conditions [18]. In particular, drought and heat threaten food security. Thus, discovery and identification of stress-related genes is a very important topic. Proteomic analysis of the upland rice IRAT 109 at the seedling stage under drought stress was undertaken in our previous study, and 9 OsHsps involved in drought stress were identified [19]. To obtain additional information on the 9 OsHsp genes, the present study fo- cused on an expression profile analysis of the OsHsp genes at different development stages throughout the life cycle of rice and under different abiotic stress and phytohormone treatments.

\section{Materials and methods}

\subsection{Identification of the 9 OsHsp genes}

Nine OsHsps were identified from the proteomic analysis in our previous study [19]. Molecular weights and theoretical isoelectric points $(\mathrm{pI})$ were calculated with the EXPASY tool (http://www.expasy.org/tools/protparam.html). The characteristics of the genes are listed in Table 1. Cis-elements in the 1-kb upstream region of the 9 OsHsp genes were identified by analyzing the sequences in the Plant cis-acting Regulatory DNA Elements database (PLACE, http://www.dna. affrc.go.jp/PLACE/signalscan.html).

\subsection{Genome-wide expression analysis of OsHsps in 2 rice cultivars}

Expression profile data for OsHsps in 27 tissues of Minghui 63 and Zhenshan 97 were extracted from the CREP database [20]. Twenty-seven tissues (Table 2) from throughout the rice life cycle were selected. Expression values of each gene were logarithmized and cluster analyses were performed in $R$ with euclidean distances and complete linkage hierarchical clustering.

Expression data was analyzed with Student's $t$-test to identify differentially expressed genes. The genes that were up- or down-regulated by more than 2 -fold and with $P<$ 0.05 were considered to be differentially expressed.

\subsection{Expression analysis of the 9 OsHsp genes under abiotic stresses}

Seeds of rice cv. IRAT109 were imbibed for $3 \mathrm{~d}$ in water, sown on moist paper and germinated in the dark at $25^{\circ} \mathrm{C}$. Germinated seeds were planted in a plastic container with

Table 1 Characteristics of the 9 OsHsp proteins in rice

\begin{tabular}{|c|c|c|c|c|}
\hline Gene name & Molecular weight (Dalton) & TIGR locus & Isoelectric point & Family \\
\hline OsHspl8.03 & 18030.82 & LOC_Os01g08860.1 & 5.61 & sHsp \\
\hline OsHsp24.15 & 24153.29 & LOC_Os02g52150.1 & 7.96 & sHsp \\
\hline OsHsp71.10 & 71100.37 & LOC_Os03g16860.1 & 5.1 & Hsp70 \\
\hline OsHsp71.18 & 71184.71 & LOC_Os11g47760.1 & 5.1 & Hsp70 \\
\hline OsHsp72.57 & 72565.29 & LOC_Os03g02260.1 & 5.71 & Hsp70 \\
\hline OsHsp72.90 & 72897.5 & LOC_Os02g53420.1 & 5.49 & Hsp70 \\
\hline OsHsp 85.88 & 85878.03 & LOC_Os08g38086.1 & 4.98 & Hsp90 \\
\hline OsHsp93.04 & 93044.79 & LOC_Os06g50300.1 & 4.89 & Hsp90 \\
\hline OsHsp108.99 & 108985.34 & LOC_Os03g31300.1 & 6.25 & Hsp100 \\
\hline
\end{tabular}


Table 2 Tissues selected from different developmental stages throughout the life cycle of rice

\begin{tabular}{|c|c|c|}
\hline $\begin{array}{c}\text { Sample } \\
\text { No. }\end{array}$ & Developmental stage & Tissues \\
\hline 1 & $3 \mathrm{~d}$ after sowing & embryo and radicle \\
\hline 2 & $15 \mathrm{~d}$ after subculture & calli \\
\hline 3 & screening stage & calli \\
\hline 4 & $5 \mathrm{~d}$ after regeneration & calli \\
\hline 5 & $72 \mathrm{~h}$ after imbibition & seed \\
\hline 6 & trefoil stage & seedling \\
\hline 7 & seedling with 2 tillers & shoot \\
\hline 8 & seedling with 2 tillers & root \\
\hline 9 & $5 \mathrm{~d}$ before heading & stem \\
\hline 10 & $5 \mathrm{~d}$ before heading & flag leaf \\
\hline 11 & heading stage & stem \\
\hline 12 & heading stage & panicle \\
\hline 13 & $14 \mathrm{~d}$ after heading & flag leaf \\
\hline 14 & $\begin{array}{l}\text { young panicle at stage } 3 \text { (secondary branch } \\
\text { primordium differentiation stage) }\end{array}$ & leaf \\
\hline 15 & young panicle at stage 3 & sheath \\
\hline 16 & young panicle at stage 3 & panicle \\
\hline 17 & $\begin{array}{l}\text { young panicle at stage } 4 \text { (pistil/stamen } \\
\text { primordium differentiation stage) }\end{array}$ & panicle \\
\hline 18 & $\begin{array}{l}\text { young panicle at stage } 5 \text { (pollen-mother } \\
\text { cell formation stage) }\end{array}$ & panicle \\
\hline 19 & $4-5 \mathrm{~cm}$ young panicle & leaf \\
\hline 20 & $4-5 \mathrm{~cm}$ young panicle & sheath \\
\hline 21 & $4-5 \mathrm{~cm}$ young panicle & panicle \\
\hline 22 & $1 \mathrm{~d}$ before flowering & hull \\
\hline 23 & $1 \mathrm{~d}$ before flowering & stamen \\
\hline 24 & $3 \mathrm{~d}$ after pollination & spikelet \\
\hline 25 & $7 \mathrm{~d}$ after pollination & endosperm \\
\hline 26 & $14 \mathrm{~d}$ after pollination & endosperm \\
\hline 27 & $21 \mathrm{~d}$ after pollination & endosperm \\
\hline
\end{tabular}

Hoagland nutrient solution, and were incubated in a growth

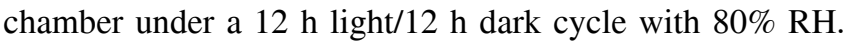
The trefoil-stage seedlings were subjected to the following five treatments. To simulate drought stress, salt stress, and for abscisic acid (ABA) treatment, seedlings were irrigated with Hoagland liquid culture containing $20 \%$ polyethyleneglycol 6000 (PEG), $150 \mathrm{mmol} / \mathrm{L} \mathrm{NaCl}$, and $100 \mu \mathrm{mol} / \mathrm{L} \mathrm{ABA}$, respectively. For cold and heat shock treatments, seedlings were exposed to 4 and $42^{\circ} \mathrm{C}$. Samples were collected after treatment exposure for $0,3,6,12$, and $24 \mathrm{~h}$ and after recovery for $24 \mathrm{~h}$. Ten shoots were combined as one biological replicate and each treatment consisted of three repeats.

Samples were ground in liquid nitrogen using a mortar and pestle. Total RNA was extracted using TRIzol reagent (Invitrogen) according to the manufacturer's instructions. First-strand cDNA was synthesized using the SuperScript First-strand Synthesis Kit (Invitrogen, USA). Real-time PCR was performed in an optical 96-well plate, including $12.5 \mu \mathrm{L}$ $2 \times$ SYBR Green Master Mix Reagent (TaKaRa), $1 \mu \mathrm{L}$ cDNA samples, and $0.2 \mathrm{mmol} / \mathrm{L}$ of each gene-specific primer, in a final volume of $25 \mu \mathrm{L}$, using the following thermal cycling procedure: $50^{\circ} \mathrm{C}$ for $2 \mathrm{~min}, 95^{\circ} \mathrm{C}$ for $10 \mathrm{~min}$; 40 or
45 cycles of $95^{\circ} \mathrm{C}$ for $30 \mathrm{~s} ; 60^{\circ} \mathrm{C}$ for $30 \mathrm{~s}$; and $72^{\circ} \mathrm{C}$ for 1 min. Disassociation curve analysis was performed as follows: $95^{\circ} \mathrm{C}$ for $15 \mathrm{~s} ; 60^{\circ} \mathrm{C}$ for $20 \mathrm{~s}$; and $95^{\circ} \mathrm{C}$ for $15 \mathrm{~min}$. The actinl gene was used as an endogenous control to normalize variance in the quality of RNA and the amount of cDNA used. Primers were designed according to the sequences within the boundaries defined by the cDNA ends (Table S1) and synthesized by Invitrogen.

\section{Results}

\subsection{Identification of the 9 OsHsp genes}

In the proteomic analysis, 13 spots representing 9 OsHsp proteins were up-regulated under drought stress. These OsHsps were designated as OsHsp18.03, OsHsp24.15, OsHsp71.10, OsHsp71.18, OsHsp72.57, OsHsp72.90, OsHsp85.88, OsHsp93.04 and OsHsp108.99, which were divided into 4 classes according to the molecular weight. Two genes (OsHsp18.03 and OsHsp24.15) belong to the sHsp family, 4 OsHsps (OsHsp71.10, OsHsp71.18, OsHsp72.57 and Os$H s p 72.90)$ are part of the OsHsp70 family, 2 genes (OsHsp85.88 and OsHsp93.04) belong to the OsHsp90 family, and OsHsp108.99 belongs to the OsHsp100 family.

\subsection{Cis-elements in the upstream region of the 9 OsHsp genes}

A search of the PLACE database with the 1-kb upstream region of the 9 OsHsp genes as queries found at least 19 previously identified putative stress-responsive or organspecific cis-elements in the promoter regions of the $O s H s p$ genes (Table S2). These cis-elements include the heat shockresponsive CCAATBOX [21], the ABA-responsive ABRELATERD1, the dehydration-responsive ACGTATERD1 and MYBCORE [22,23], the light-regulated GT1CONSENSUS and IBOXCORE [24], and the defense-responsive WBOXATNPR1 [25]. The dehydration-responsive ACGTATERD1 was the most frequent stress-related cis-element, with 10 and 14 found in $O s H s p 72.9$ and OsHsp93.4, respectively.

\subsection{Expression analysis of OsHsp genes in different tissues}

The corresponding probes for all of the 9 OsHsp genes were included on the Crep chip data. The average signal values of the 9 OsHsp genes are listed in Table S3.

Hierarchical cluster analysis based on the signal values showed that the OsHsp genes had very diverse expression patterns (Figure 1). The 9 OsHsp genes were expressed in at least one developmental stage or tissue. Hierarchical clustering, which was based on the mean log-transformed intensity value of the genes, enabled the expression profiles to be divided into two major groups. Group I contained 4 genes (OsHsp93.04, OsHsp71.10, OsHsp85.88 and OsHsp108.99). 


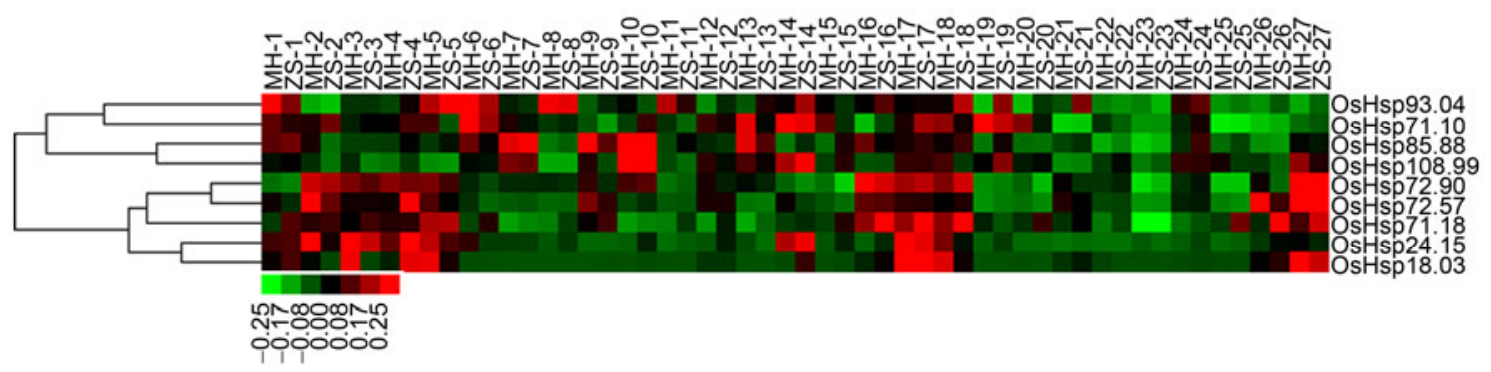

Figure 1 Hierarchical clustering display of $O s H s p$ family genes in rice based on the average signal values in 27 tissues. Numbers refer to: 1 , embryo and radicle after germination; 2, calli, $15 \mathrm{~d}$ after subculture; 3 , calli, screening stage; 4 , calli, $5 \mathrm{~d}$ after regeneration; 5 , seed, $72 \mathrm{~h}$ after imbibition; 6 , root and leaf at three-leaf stage; 7, shoot, seedling with 2 tillers; 8, root, seedling with 2 tillers; 9 , stem, $5 \mathrm{~d}$ before heading; 10 , flag leaf, $5 \mathrm{~d}$ before heading; 11 , stem, heading stage; 12 , panicle, heading stage; 13 , flag leaf, $14 \mathrm{~d}$ after heading; 14 , leaf, young panicle at stage $3 ; 15$, sheath, young panicle at stage 3 ; 16 , young panicle at stage $3 ; 17$, young panicle at stage $4 ; 18$, young panicle at stage $5 ; 19$, leaf, $4-5 \mathrm{~cm}$ young panicle; 20 , sheath, 4-5 cm young panicle; 21 , panicle, 4-5 cm young panicle; 22, hull, $1 \mathrm{~d}$ before flowering; 23, stamen, $1 \mathrm{~d}$ before flowering; 24, spikelet, $3 \mathrm{~d}$ after pollination; 25, endosperm, $7 \mathrm{~d}$ after pollination; 26, endosperm, $14 \mathrm{~d}$ after pollination; 27, endosperm, $21 \mathrm{~d}$ after pollination. Each number comprises three parallel lines that indicate Minghui 63 (MH) and Zhenshan 97 (ZS), respectively. The colour scale (representing average signal values) is shown at the bottom.

Transcripts of both OsHsp93.04 and OsHsp71.10 were more frequent in the embryo, leaf and panicle, and OsHsp71.10 was also highly expressed in the root. High expression levels of OsHsp85.88 and OsHsp108.99 were detected in the embryo, leaf, stem, panicle and mature endosperm $(21 \mathrm{~d}$ after pollination).

The expression patterns of the genes in Group II differed from those of Group I. All 5 genes were highly expressed in calli, the panicle and endosperm. Up-regulation of OsHsp72.90 was detected $21 \mathrm{~d}$ after pollination in endosperm, and transcripts of OsHsp72.59 were up-regulated in the endosperm at 14 and $21 \mathrm{~d}$ after pollination. Expression levels of OsHsp71.18 were lower in Minghui 63 than those in Zhenshan 97. In addition, gradual up-regulation of OsHsp71.18 was detected in the endosperm from $7 \mathrm{~d}$ after pollination to $21 \mathrm{~d}$ after pollination. OsHsp24.15 was predominantly expressed in calli, the embryo, leaf (young panicle at secondary branch primordium differentiation stage), young panicle at stage 4 (pistil/stamen primordium differentiation stage) and stage 5 (pollen mother cell formation stage), and moderately expressed in the endosperm. OsHsp18.03 was expressed preferentially in calli, the young panicle at stage 4 (pistil/stamen primordium differentiation stage), and was gradually up-regulated in the endosperm from $7 \mathrm{~d}$ after pollination to $21 \mathrm{~d}$ after pollination.

Overall, the $O s H s p$ genes in Group I showed a higher expression level in vegetative tissues, and fewer transcripts were detected in calli and the endosperm compared to those of OsHsps in Group II. The expression patterns were almost identical between Minghui 63 and Zhenshan 97, except for the expression levels.

\subsection{Expression profiles of the 9 OsHsps under phyto- hormone treatments at trefoil seedling stage}

The responses of the 9 OsHsp genes to gibberellin3 (GA3), Naphthaleneacetic Acid (NAA) and Kinetin (KT) were also based on the CREP chip data. Five genes were responsive to all three phytohormones. Three OsHsp genes (OsHsp71.10,
OsHsp71.18 and OsHsp93.04) were down- regulated under the GA3, NAA and KT treatments, whereas two genes (OsHsp85.88 and OsHsp108.99) were up-regu- lated in response to these hormones. The hierarchical cluster display of the 9 genes in the hormonal treatments in Minghui 63 and Zhenshan 97 is shown in Figure 2, and the average signal values are presented in Table S4.

Because the 9 OsHsp genes were identified by proteomic analysis of rice IRAT 109 leaf samples at the seedling stage under drought stress, IRAT109 seedlings were treated with ABA at the three-leaf stage. Interestingly, all 9 genes were significantly up-regulated after ABA treatment for $12 \mathrm{~h}$. However, the expression levels decreased back to the levels of non-stressed samples after the stress was released for $24 \mathrm{~h}$ (Figure 3).

\subsection{Expression patterns of the 9 OsHsps under abiotic stresses}

Numerous abiotic stresses are known to trigger alteration in

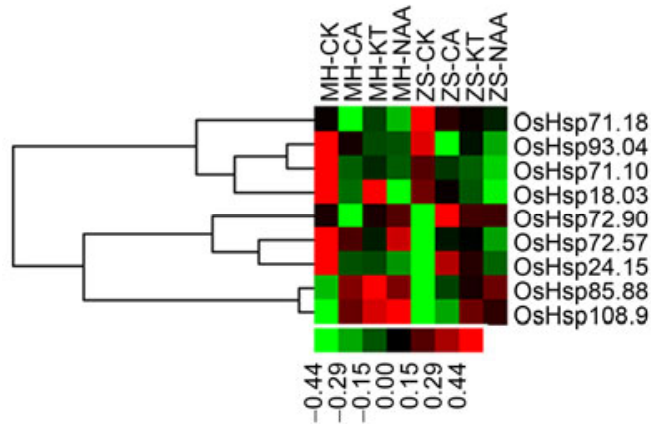

Figure 2 Expression profile of $O s H s p$ family genes under different hormonal stress conditions (NAA, GA3 and KT) in Minghui 63 and Zhenshan 97. The colour scale (representing average signal values) is shown at the bottom. MH-CK (Minghui 63, control), MH-GA3 (Minghui 63, GA3), MH-KT (Minghui 63, KT), MH-NAA (Minghui 63, NAA), ZH-CK (Zhenshan 97, control), ZH-GA3 (Zhenshan 97, GA3) ZH-KT (Zhenshan 97, KT) and ZH-NAA (Zhenshan 97, NAA). 
(a)

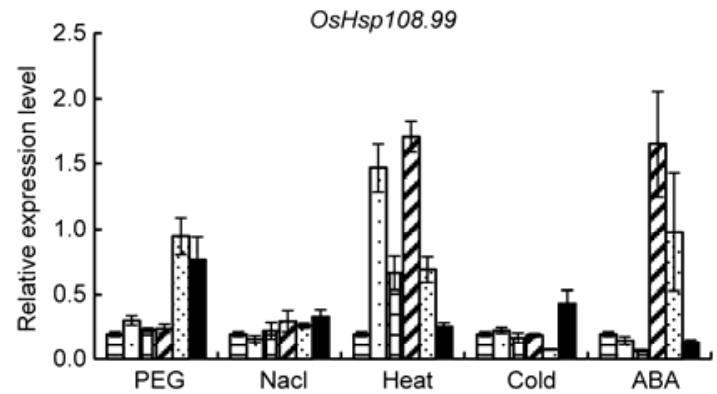

(b)

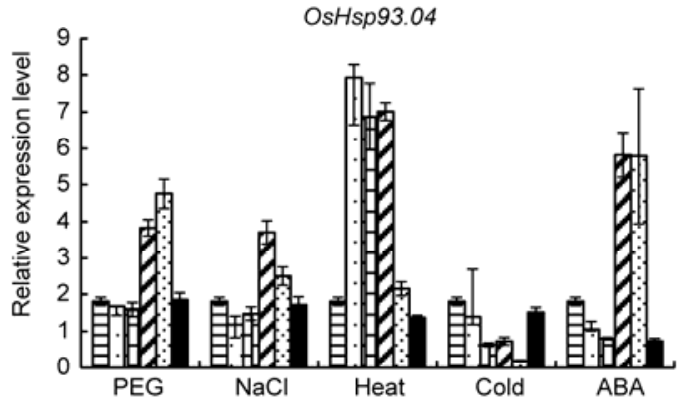

(c)
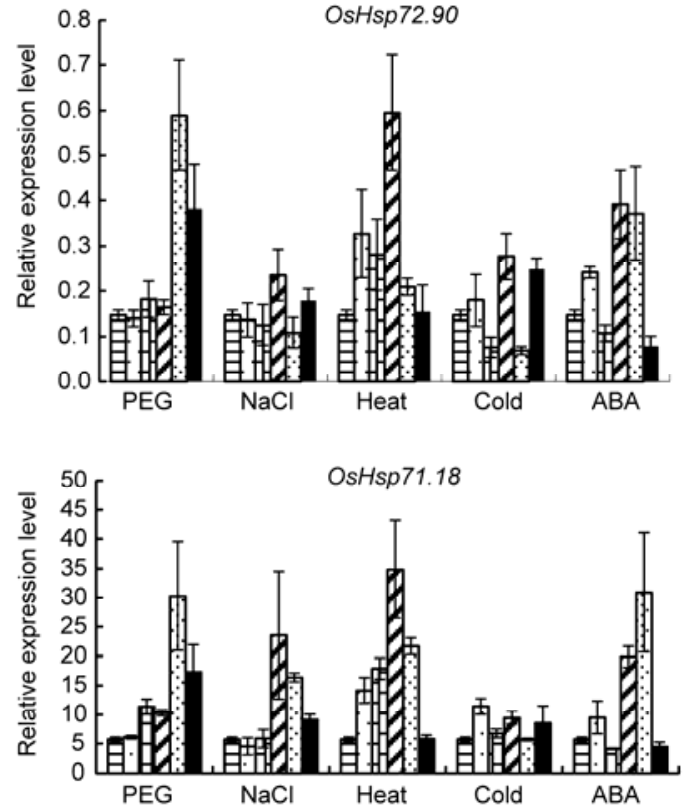

(d)

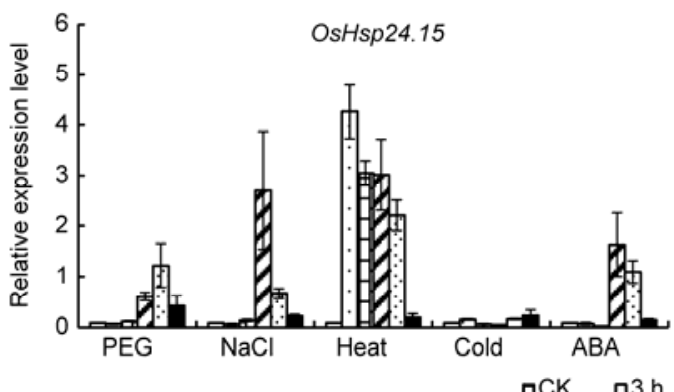

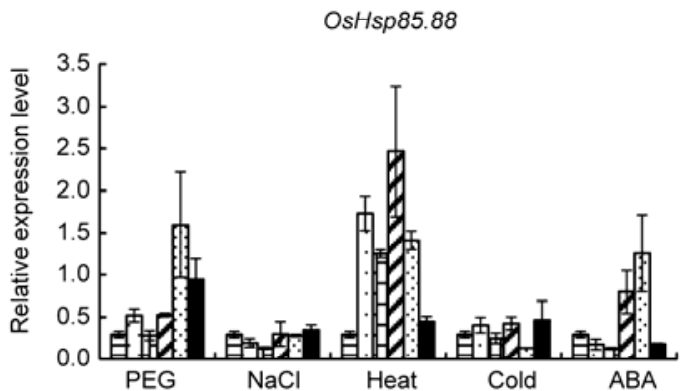

OsHsp72.57

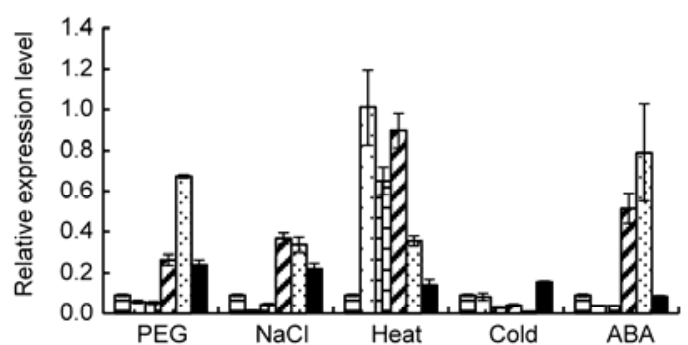

OsHsp71.10
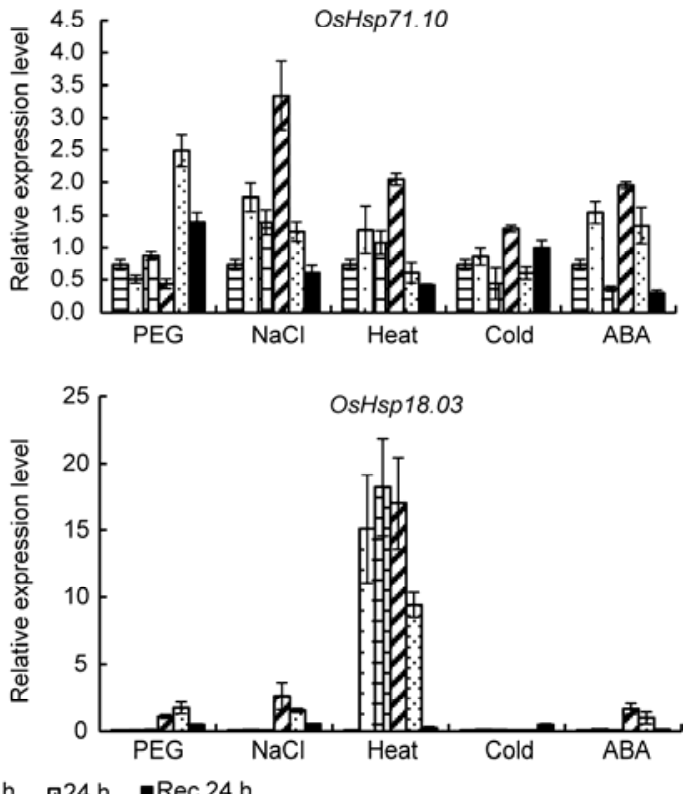

Figure 3 Relative quantification by real-time PCR analysis of the expression levels of OsHsp genes in rice IRAT109 in response to abiotic stress treatments. Rice Actinl was used as the internal control. The bars are means of the relative fold change of three biological and two technical replicates obtained by real-time PCR. The standard errors of the biological replicates are shown as error bars. Relative expression levels are shown for (a) OsHsp108.99, which belongs to the $O s H s p 100$ family; (b) the two OsHsp genes in the OsHsp9O family; (c) the four OsHsp genes in the $O s H s p 70$ family; and (d) the two $O s H s p$ genes in the $s H s p$ family. 
the transcription of $H s p$ genes. Therefore, we further checked the expression profiles of the 9 OsHsp genes under diverse abiotic stress treatments using real-time PCR. Under dehydration stress, transcript levels of the 9 OsHsp genes were enhanced. Four genes (OsHsp93.04, OsHsp72.57, OsHsp24.15 and OsHsp18.03) were up-regulated after 12-h treatment with PEG, and a high level of transcripts of the other five genes was detected after 24-h treatment. After recovery for $24 \mathrm{~h}$, transcript levels of most of the OsHsp genes decreased to the level prior to stress treatment.

Significantly increased levels of the 9 OsHsp genes were detected after $3 \mathrm{~h}$ at $42^{\circ} \mathrm{C}$, which indicated these genes are quickly and sharply induced by heat shock stress. This was especially true for the genes OsHsp24.15 and OsHsp18.03, which were up-regulated by more than 48 - and 227-fold, respectively.

Salt stress caused up-regulation of 6 genes (OsHsp93.04, OsHsp71.10, OsHsp71.18, OsHsp72.57, OsHsp24.15 and OsHsp18.03). However, cold stress caused down-regulation of OsHsp93.04 and OsHsp72.57.

\section{Discussion}

\subsection{OsHsp genes may be involved in seed development}

Plant Hsps are expressed during an intriguing array of developmental processes, which indicates they may have important roles in the absence of stresses [26]. The endosperm in monocot seeds plays a very important role in seed development and can be the principal nutrient storage tissue in seeds. The genes in OsHsp Group II (OsHsp72.90, OsHsp 72.57, OsHsp71.18, OsHsp24.15 and OsHsp18.03) showed high expression in the endosperm, indicating that $O s H s p$ genes may play important roles in rice seed development.

In plants, sHsps are generally undetectable in vegetative tissues in the absence of stress, but are expressed during specific stages of plant development, and sHsp expression during seed development is the most extensively characterized example of this non-stress regulation [27]. sHsps accumulated at mid-maturation and were abundant throughout the late maturation period and in the dry seed in Arabidopsis [26], pea [28], and sunflower [29].

AtHsp101 is a component of mature seeds and regulates seed development in the absence of stress, in a pattern similar to that seen for Late Embryogenesis Abundant (LEA) proteins and small Hsps; protein accumulates during midmaturation and is stored in the dry seed [29,30].

The mechanisms that regulate Hsp expression during seed maturation remain largely unknown. Using a mutant approach, a genetic framework in which HsfA9 operates as a specialized heat-shock transcription factor (Hsf) for the developmental expression of Hsp genes during seed maturation was established, which showed that ABI3 could activate the HsfA9 promoter, whereas HsfA9 in turn was shown to be a potent activator on the promoters of Hsp genes [31].

\subsection{Function of OsHsps under drought and heat stress}

Drought and heat stress are among the abiotic factors that cause the most severe damage to plant crops. In nature, individual stresses, such as drought and high temperature, usually do not occur separately. A combination of drought and heat shock resulted in the closure of stomata, suppression of photosynthesis, enhancement of respiration, and increased leaf temperature. To counter the effects of such stress, plants express a variety of defense genes such as heat shock proteins [32].

The 9 OsHsps analyzed in the present study were identified in a proteomic analysis of rice leaf samples at the seedling stage under drought stress in our previous study [19]. The transcription levels of all 9 OsHsp genes were significantly enhanced both under dehydration and heat stress, which showed they may play roles in drought and heat stress.

In the cis-element analysis of the 9 OsHsp genes, ciselements related to heat shock and dehydration were observed. It is well known that the heat-inducible binding of Hsfs to the heat-shock element (HSE) promoter sequences control the expression of Hsp genes during stress and development $[33,34]$. The analysis carried out in the present study showed that promoters of the 9 OsHsp genes harbor a minimum of one module of a putative HSE. The dehydration-responsive elements ACGTATERD1 and MYBCORE existed in the promoter of the 9 OsHsp genes. ACGTATERD1 and MYBCORE are important cis-elements for ERD1 and MYB proteins, respectively, involved in the regulation of stress-responsive genes [22,23].

In Arabidopsis, the expression level of At-Hsp17.4 was decreased in mutant lines and resulted in a seed desiccation-intolerance phenotype, and At-Hsp17.6A was induced by heat and osmotic stress [27,35]. In rice, tolerance to drought stress was enhanced in transgenic rice plants overexpressing a small heat-shock protein, sHsp17.7, and transgenic seedlings showing higher expression levels of sHsp 17.7 protein could regrow after resumption of watering [17]. In tobacco, transgenic tobacco plants producing elevated levels of the tobacco Hsp70, NtHsp70-1, exhibited tolerance to water stress, as indicated by the higher leaf water content in the transgenic plants compared to that of the wild type [36]. In addition, seven Hsps were induced in response to a combination of drought and heat shock. These included cytosolic Hsp90, Hsp70, and Hsp100, and sHsps (cytosolic, mitochondrial, and chloroplastic). Furthermore, the induction of Hsps was higher in response to drought and heat shock compared with heat shock or drought individually [37]. In maize, Hsp70 and sHsps (sHsp17.2, sHsp17.4 and sHsp26) are responsive to a combination of drought and heat stress [12,38]. Four Hsp genes (Hsp17.8, Hsp26.3, Hsp70 and Hsp101b) in Triticum aestivum plants were monitored via one-step RT-PCR and SDS-PAGE analysis in plants subjected to water deprivation or high temperature individually and in combination. The combination of drought 
and heat induced Hsp expression more effectively than the individually applied stresses and the induction of Hsps was greater in the drought-tolerant wheat cultivar Katya than in the drought-sensitive cultivar Sadovo [39,40].

\subsection{OsHsp genes may be involved in ABA-regulated pathways}

ABA regulates many plant responses to environmental stress. The greatest increase in ABA concentration is observed in response to drought stress, and temperature also has an effect on endogenous ABA levels [41].

The 9 OsHsp genes identified in the present study were up-regulated under PEG and heat stress as well as in response to ABA treatment. Moreover, ABRELATERD1, a cis-acting element involved in the ABA function, was present in all $9 O s H s p$ genes, which indicated they may be associated with the key roles of ABA in plant metabolism.

$H s p$ genes regulated by ABA have been reported in several plant species, such as maize, tobacco, wheat and rice [12,36,42-44]. Results of studies with an ABA-deficient mutant indicated that endogenous ABA regulated Hsp70 synthesis in the leaves and roots of maize seedlings exposed to drought, heat, and combined drought and heat stress, which implied that $\mathrm{ABA}$ may improve plant tolerance to stress by increasing $\mathrm{Hsp} 70$ expression. In addition, $\mathrm{ABA}$ is involved in the enhancement of enzyme activities and $\mathrm{H}_{2} \mathrm{O}_{2}$ production in leaves and roots subjected to a number of stress treatments [12].

In summary, analysis of the tissue and phytohormone expression profiles of the 9 OsHsp genes has improved the functional dissection of rice $H s p$ genes. Elucidation of the precise role of each $O s H s p$ gene, however, requires the use of other experimental approaches including, for example, overexpression and/or RNAi strategies.

This work was supported by the National Program on Research and Development of Transgenic Plants of China (2008ZX08001-001), the Key Project of Shanghai Municipal Agriculture Commission (2009-6-4-2), and the Shanghai Municipal Science and Technology Commission, Shanghai Postdoctoral Fund (10R21420400).

1 Reddy P S, Mallikarjuna G, Kaul T, et al. Molecular cloning and characterization of gene encoding for cytoplasmic Hsc70 from Pennisetum glaucum may play a protective role against abiotic stresses. Mol Gen Genet, 2010, 283: 243-254

2 Queitsch C, Hong S W, Vierling E, et al. Heat shock protein 101 plays a crucial role in thermotolerance in Arabidopsis. Plant Cell, 2000, 12: 479-492

3 Liu D, Zhang X, Cheng Y, et al. rHsp90 gene expression in response to several environmental stresses in rice (Oryza sativa L.). Plant Physiol Biochem, 2006, 44: 380-386

4 Takahashi A, Casais C, Ichimura K, et al. HSP90 interacts with RAR1 and SGT1 and is essential for RPS2-mediated disease resistance in Arabidopsis. Proc Natl Acad Sci USA, 2003, 100: 11777-11782

5 Yamada K, Fukao Y, Hayashi M, et al. Cytosolic HSP90 regulates the heat shock response that is responsible for heat acclimation in Arabidopsis thaliana. J Biol Chem, 2007, 282: 37794-37804
$6 \mathrm{Xu} \mathrm{X}$, Song H, Zhou Z, et al. Functional characterization of AtHsp90.3 in Saccharomyces cerevisiae and Arabidopsis thaliana under heat stress. Biotechnol Lett, 2010, 32: 979-987

7 Reddy P S, Thirulogachandar V, Vaishnavi C S, et al. Molecular characterization and expression of a gene encoding cytosolic Hsp90 from Pennisetum glaucum and its role in abiotic stress adaptation. Gene, 2011, 474: 29-38

8 Lin B L, Wang J S, Liu H C, et al. Genomic analysis of the Hsp70 superfamily in Arabidopsis thaliana. Cell Stress Chaperones, 2001, 6: 201-208

9 Jungkunz I, Link K, Vogel F, et al. AtHsp70-15 deficient Arabidopsis plants are characterized by reduced growth, a constitutive cytosolic protein response and enhanced resistance to TuMV. Plant J, 2011, doi: 10.1111/j.1365-313X.2011.04558.x

$10 \mathrm{Su}$ P H, Li H M. Arabidopsis stromal 70-kD heat shock proteins are essential for plant development and important for thermotolerance of germinating seeds. Plant Physiol, 2008, 146: 1231-1241

11 Duan Y H, Guo J, Ding K, et al. Characterization of a wheat HSP7O gene and its expression in response to stripe rust infection and abiotic stresses. Mol Biol Rep, 2010, 38: 301-307

$12 \mathrm{Hu}$ X, Liu R, Li Y, et al. Heat shock protein 70 regulates the abscisic acid-induced antioxidant response of maize to combined drought and heat stress. Plant Growth Regul, 2009, 60: 225-235

13 Montero-Barrientos M, Hermosa R, Cardoza R E, et al. Transgenic expression of the Trichoderma harzianum hsp70 gene increases Arabidopsis resistance to heat and other abiotic stresses. J Plant Physiol, 2010, 167: 659-665

14 Qi Y, Wang H, Zou Y, et al. Over-expression of mitochondrial heat shock protein 70 suppresses programmed cell death in rice. FEBS Lett, 2011, 585: 231-239

15 Al-Whaibi M H. Plant heat-shock proteins: A mini review. J King Saud Univ Sci, 2010, doi: 10.1016/j.jksus.2010.06.022

16 Sun W N, Montagu M V, Verbruggen N. Small heat shock proteins and stress tolerance in plants. Biochim Biophys Acta, 2002, 1577: $1-7$

17 Sato Y, Yokoya S. Enhanced tolerance to drought stress in transgenic rice plants over-expressing a small heat-shock protein, sHSP17.7. Plant Cell Rep, 2008, 27: 329-334

18 Zhang Q. Strategies for developing Green Super Rice. Proc Natl Acad Sci USA, 2007, 104: 16402-16409

19 Shu L, Lou Q, Ma C, et al. Genetic, proteomic, and metabolic analysis of the regulation of energy storage in rice seedlings in response to drought. Proteomics, 2011, doi: 10.1002/pmic.201000485

20 Wang L, Xie W, Chen Y, et al. A dynamic gene expression atlas covering the entire life cycle of rice. Plant J, 2010, 61: 752-766

21 Rieping M, Schoffl F. Synergistic effect of upstream sequences, CCAAT box elements, and HSE sequences for enhanced expression of chimaeric heat shock genes in transgenic tobacco. Mol Gen Genet, 1992, 231: 226-232

22 Simpson S D, Nakashima K, Narusaka Y, et al. Two different novel cis-acting elements of erd1, a clpA homologous Arabidopsis gene function in induction by dehydration stress and dark-induced senescence. Plant J, 2003, 33: 259-270

23 Urao T, Yamaguchi-Shinozaki K, Urao S, et al. An Arabidopsis myb homolog is induced by dehydration stress and its gene product binds to the conserved MYB recognition sequence. Plant Cell, 1993, 5: 1529-1539

24 Terzaghi W B, Cashmore A R. Light-regulated transcription. Annu Rev Plant Physiol Plant Mol Biol, 1995, 46: 445-474

25 Yu D, Chen C, Chen Z. Evidence for an important role of WRKY DNA binding proteins in the regulation of NPR1 gene expression. Plant Cell, 2001, 13: 1527-1540

26 Wehmeyer N, Hernandez L D, Finkelstein R R, et al. Synthesis of small heat-shock proteins is part of the developmental program of late seed maturation. Plant Physiol, 1996, 112: 747-757

27 Wehmeyer N, Vierling E. The expression of small heat shock proteins in seeds responds to discrete developmental signals and suggests a general protective role in desiccation tolerance. Plant Physiol, 2000, 122: 1099-1108 
28 DeRocher A, Vierling E. Developmental control of small heat shock protein expression during pea seed maturation. Plant J, 1994, 5: 93-102

29 Coca M, Almoguera C, Jordano J. Expression of sunflower low-molecular-weight heat-shock proteins during embryogenesis and persistence after germination: Localization and possible functional implications. Plant Mol Biol, 1994, 25: 479-492

30 Hong S W, Vierling E. Hsp101 is necessary for heat tolerance but dispensable for development and germination in the absence of stress. Plant J, 2001, 27: 25-35

31 Kotak S, Vierling E, Baumlein H, et al. A novel transcriptional cascade regulating expression of heat stress proteins during seed development of Arabidopsis. Plant Cell, 2007, 19: 182-195

32 Vierling E. The roles of heat shock proteins in plants. Annu Rev Plant Physiol Plant Mol Biol, 1991, 42: 579-620

33 Almoguera C, Prieto-Dapena P, Jordano J. Dual regulation of a heat shock promoter during embryogenesis: Stage-dependent role of heat shock elements. Plant J, 1998, 13: 437-446

34 Volkov R A, Panchuk I I, Mullineaux P M, et al. Heat stress-induced $\mathrm{H}(2) \mathrm{O}(2)$ is required for effective expression of heat shock genes in Arabidopsis. Plant Mol Biol, 2006, 61: 733-746

35 Sun W, Bernard C, van de Cotte B, et al. At-HSP17.6A, encoding a small heat-shock protein in Arabidopsis, can enhance osmotolerance upon overexpression. Plant J, 2001, 27: 407-415

36 Cho E K, Hong C B. Over-expression of tobacco NtHSP70-1 contributes to drought-stress tolerance in plants. Plant Cell Rep, 2006, 25 :
349-358

37 Rizhsky L, Liang H, Mittler R. The combined effect of drought stress and heat shock on gene expression in tobacco. Plant Physiol, 2002, 130: 1143-1151

$38 \mathrm{Hu} \mathrm{X}, \mathrm{Li} \mathrm{Y}, \mathrm{Li} \mathrm{C}$, et al. Characterization of small heat shock proteins associated with maize tolerance to combined drought and heat stress. J Plant Growth Regul, 2010, 29: 455-464

39 Grigorova B, Vaseva I, Demirevska K, et al. Combined drought and heat stress in wheat: Changes in some heat shock proteins. Biol Plant, 2011, 55: 105-111

40 Grigorova B, Vaseva I, Demirevska K, et al. Expression of selected heat shock proteins after individually applied and combined drought and heat stress. Acta Physiol Plant, 2011, 33: 2041-2049

41 Daie J, Campbell W F. Response of tomato plants to stressful temperatures: Increase in abscisic acid concentrations. Plant Physiol, 1981, 67: 26-29

42 Heikkila J J, Papp J E, Schultz G A, et al. Induction of heat shock protein messenger RNA in maize mesocotyls by water stress, abscisic acid, and wounding. Plant Physiol, 1984, 76: 270-274

43 Campbell J L, Klueva N Y, Zheng H G, et al. Cloning of new members of heat shock protein HSP101 gene family in wheat (Triticum aestivum (L.) Moench) inducible by heat, dehydration, and $\mathrm{ABA}(1)$. Biochim Biophys Acta, 2001, 1517: 270-277

44 Zou J, Liu A, Chen X, et al. Expression analysis of nine rice heat shock protein genes under abiotic stresses and ABA treatment. J Plant Physiol, 2009, 166: 851-861

Open Access This article is distributed under the terms of the Creative Commons Attribution License which permits any use, distribution, and reproduction in any medium, provided the original author(s) and source are credited.

\section{Supporting Information}

Table S1 Primers used in the real-time PCR analysis

Table S2 Number of cis-elements in the 1-kb upstream region of OsHsp genes

Table S3 Average signal values of the nine OsHsp genes expressed in 27 tissues for Minghui 63 and Zhenshan 97

Table S4 Average signal values of the nine OsHsp genes under the hormonal treatments for Minghui 63 and Zhenshan 97

The supporting information is available online at csb.scichina.com and www.springerlink.com. The supporting materials are published as submitted, without typesetting or editing. The responsibility for scientific accuracy and content remains entirely with the authors. 